\title{
Identification of a novel subgroup of uncultured gammaproteobacterial glycogen-accumulating organisms in enhanced biological phosphorus removal sludge
}

\author{
Jeong Myeong Kim, ${ }^{1}$ Hyo Jung Lee, ${ }^{1}$ Dae Sung Lee, ${ }^{2}$ Kangseok Lee ${ }^{1}$ \\ and Che Ok Jeon ${ }^{1}$ \\ ${ }^{1}$ School of Biological Sciences, Chung-Ang University, HeukSeok-Dong, Dongjak-Gu, Seoul, \\ 156-756, Republic of Korea \\ ${ }^{2}$ Department of Environmental Engineering, Kyungpook National University, Sankyuk-dong, Buk-gu, \\ Daegu 702-701, Republic of Korea
}

Correspondence

Che Ok Jeon

cojeon@cau.ac.kr
Received 25 April 2011

Revised 15 May 2011

Accepted 16 May 2011
The presence of glycogen-accumulating organisms (GAO) has been hypothesized to be a cause of deterioration in enhanced biological phosphorus removal (EBPR) processes due to their abilities to out-compete polyphosphate-accumulating organisms (PAO). Based on $16 \mathrm{~S}$ rRNA gene sequences, new members of uncultured gammaproteobacterial GAO (GB) were identified from sludge samples of a lab-scale sequencing batch reactor used for EBPR. The new GB formed a phylogenetic lineage (GB8) clearly distinct from the previously reported seven GB subgroups. Because the new GB8 members were not targeted by the known fluorescence in situ hybridization (FISH) oligonucleotide probes, a GB8-specific FISH probe (GB429) and a new FISH probe (GB742) targeting all eight GB subgroups were designed, and the phenotypic properties of the new GB8 members were investigated. FISH and microautoradiography approaches showed that GB429-targeted cells (GB8) were large coccobacilli (2-4 $\mu \mathrm{m}$ in size) with the ability to take up acetate under anaerobic conditions, but unable to accumulate polyphosphate under the subsequent aerobic conditions, consistent with in situ phenotypes of GB. FISH analyses on several sludge samples showed that members of GB8 were commonly detected as the majority of GB in lab- and full-scale EBPR processes. In conclusion, this study showed that members of GB8 could be a subgroup of GB with an important role in EBPR deterioration. Designs of FISH probes which hybridize with broader GB subgroups at different hierarchical levels will contribute to studies of the distributions and ecophysiologies of GB in labor full-scale EBPR plants.

\section{INTRODUCTION}

Enhanced biological phosphorus removal (EBPR) has been applied in many wastewater treatment plants to decrease the amount of phosphorus that causes eutrophication in surface waters. EBPR is achieved through the enrichment of a group of polyphosphate-accumulating organisms (PAO), generally known as 'Candidatus Accumulibacter phosphatis' (Accumulibacter), in activated-sludge systems

Abbreviations: EBPR, enhanced biological phosphorus removal; FA, formamide; FISH, fluorescence in situ hybridization; GAO, glycogenaccumulating organisms; GB, gammaproteobacterial GAO; MAR, microautoradiography; PAO, polyphosphate-accumulating organisms; PFA, paraformaldehyde; PHA, polyhydroxyalkanoates; SBR, sequencing batch reactor.

The GenBank/EMBL/DDBJ accession numbers for the 16S rRNA gene sequences retrieved in this study are HO341387-HO341392. using alternating anaerobic and aerobic cycles. According to the commonly accepted EBPR model proposed by Mino et al. (1987), short-chain volatile fatty acids, usually acetate or propionate, are taken up and converted to polyhydroxyalkanoates (PHA) by PAO during the anaerobic phase. In the subsequent aerobic phase, with oxygen as an electron acceptor and in the absence of external carbon sources, the internally stored PHA is used for cell growth and maintenance as well as polyphosphate accumulation in excess of levels normally required to satisfy the metabolic demand for growth (Oehmen et al., 2007; Seviour \& McIlroy, 2008).

Another group of micro-organisms, glycogen-accumulating organisms (GAO), often coexist with $\mathrm{PAO}$, and their presence has been hypothesized to be a cause of deterioration in EBPR processes (Cech \& Hartman, 1993; Bond et al., 1995; Liu et al., 1996; Mino et al., 1998; Seviour 
et al., 2003). Like PAO, GAO proliferate under alternating anaerobic and aerobic conditions. However, they use glycogen as their primary energy source for anaerobic volatile fatty acid uptake and PHA synthesis. Also, aerobic PHA oxidation in these organisms leads only to biomass growth and glycogen replenishment, not to polyphosphate storage (Liu et al., 1994; Satoh et al., 1994; Mino et al., 1995). These GAO are diverse and have been found in numerous lab-scale and full-scale EBPR processes. Some uncultured members of Alphaproteobacteria and Gammaproteobacteria have been proposed as GAO candidates, showing biochemical properties consistent with typical GAO metabolisms. Alphaproteobacterial GAO are closely related to the genera Sphingomonas and Defluviicoccus and have been assumed to play an important role in EBPR deterioration (Wong et al., 2004; Meyer et al., 2006; Burow et al., 2007; McIlroy et al., 2010).

Gammaproteobacterial GAO (GB) known as 'Candidatus Competibacter phosphatis' (Competibacter) have been studied intensively and are widely present in lab- and fullscale EBPR processes (Nielsen et al., 1999; Crocetti et al., 2002; Kong et al., 2002, 2006). All GB reported up to now share a similar morphology of Gram-negative large coccobacilli of $2-4 \mu \mathrm{m}$, although they are phylogenetically very diverse (Crocetti et al., 2002; Kong et al., 2002, 2006; Schroeder et al., 2009). Kong et al. (2002) showed that GB could be clustered into seven subgroups using 14 existing and 18 newly retrieved $16 \mathrm{~S}$ rRNA gene sequences. They designed a set of fluorescence in situ hybridization (FISH) probes targeting the GB subgroups at different hierarchical levels. In this study, a new subgroup (GB8) of GB that was not hybridized by the known GB-targeting FISH probes was discovered. FISH probes targeting GB8 and all GB subgroups including GB8 were designed, and the ecophysiologies and distributions of these organisms in lab- and full-scale plants were investigated using FISH and/or microautoradiography (MAR) approaches.

\section{METHODS}

Operation of the sequencing batch reactor. A cylindrical vessel with a 41 working volume was used for the sequencing batch reactor (SBR) operation. Activated sludge for the microbial inoculum was obtained from a wastewater treatment plant at the campus of POSTECH, Kyungbuk, Korea. Synthetic wastewater was used in the experiment. The feed of the synthetic wastewater contained $770 \mathrm{mg}$ sodium acetate $1^{-1}, 40 \mathrm{mg} \mathrm{NH}_{4}^{+}-\mathrm{N}^{-1}, 15 \mathrm{mg} \mathrm{PO}_{4}^{3-}-\mathrm{P} \mathrm{l}^{-1}$, and trace element mixtures (Jeon \& Park, 2000). The SBR was operated in $8 \mathrm{~h}$ anaerobic/aerobic cycles at $20{ }^{\circ} \mathrm{C}$. More details of SBR operation have been given previously (Jeon et al., 2003a). In brief, each cycle consisted of $20 \mathrm{~min}$ of anaerobic filling, $1 \mathrm{~h} 40 \mathrm{~min}$ of anaerobic reaction, $4 \mathrm{~h}$ of aerobic reaction, $90 \mathrm{~min}$ of settling and $30 \mathrm{~min}$ of decanting. Two litres of clarified supernatant was withdrawn at the end of the settling phase. Mean sludge retention time was maintained at approximately 10 days by withdrawing a small portion of sludge from the reactor at the end of the aerobic phase. Soluble orthophosphate was analysed using an ICS-1000 ion chromatograph (Dionex).
DNA extraction and phylogenetic analysis. Genomic DNA from sludge samples of the SBR was extracted using a FastDNA SPIN kit (Qbiogene) according to the manufacturer's instructions. Bacterial $16 \mathrm{~S}$ rRNA gene clone libraries were constructed as described previously (Kim et al., 2010). Briefly, modified two-step PCR amplification was used to reduce chimeric products using primers 27f and 1492r (Lane, 1991; Lu et al., 2006; Wang et al., 2008). PCR products were purified and ligated into the pCR2.1 vector using a TOPO cloning kit (Invitrogen) according to the manufacturer's instructions. After blue-white screening of the colonies, inserted 16S rRNA genes of 114 clones were amplified using vector-specific primers that flank the cloning region (Jeon et al., 2003b) and were analysed according to restriction fragment length polymorphism (RFLP) analysis after HaeIII and HhaI double digestions using previously described methods (Kim et al., 2008). All representative clones (44 clones) which showed unique fragment patterns were partially sequenced with the M13 reverse primer of the TOPO cloning kit. Resulting sequences were submitted to GenBank for BLASTN searches. If representative sequences were related to Gammaproteobacteria, the clones were more completely sequenced to determine their potential diversities. The chimeric properties of the $16 \mathrm{~S}$ rRNA gene sequences were checked using the Bellerophon program (http:// comp-bio.anu.edu.au/bellerophon/bellerophon.pl, DeSantis et al., 2006). The resulting sequences were compared with available Competibacter 16S rRNA gene sequences of Gammaproteobacteria from GenBank (http://blast.ncbi.nlm.nih.gov/Blast.cgi) and were aligned using the SILVA server (http://www.arb-silva.de, Pruesse et al., 2007). A phylogenetic tree using the neighbour-joining (NJ) algorithm was constructed by the PHYLIP software (version 3.6, Felsenstein, 2002). The resulting tree topology was evaluated using bootstrap analysis based on 1000 resamplings. Maximum-likelihood analysis was also performed using RAxML-HPC on Abe (version 7.2.6) of the Cyber-Infrastructure for Phylogenetic Research project (CIPRES, www.phylo.org; Stamatakis et al., 2005) at the San Diego Supercomputer Center.

FISH probe design and evaluation. Oligonucleotide FISH probes and competitors targeting the new GB subgroup (GB8) and whole GB subgroups were designed using the probe design tool based on comparative analyses of reliable sequences with $>1200 \mathrm{bp}$ and our clone sequences in the ARB software package (Ludwig et al., 2004). Specificities of the FISH probes were subsequently confirmed using Probe Match on the website of the Ribosome Database Project (RDP Release 10, Cole et al., 2009). The designed oligonucleotides were synthesized and labelled at the $5^{\prime}$ end with FITC or 3-iodocyanine dye (Cy3) by Thermo (Ulm, Germany). Paraformaldehyde (PFA)-fixed sludge samples were used to optimize formamide (FA) concentrations in FISH experiments because GB cannot be cultured. FA concentrations for optimum probe stringency were determined empirically by performing a series of FISH experiments at $5 \% \mathrm{FA}$ increments from $15 \%$ to $70 \%$ FA at set hybridization and wash temperatures (Hugenholtz et al., 2002). Subsequently, these designed probes were simultaneously and/or independently used with generally accepted FISH probes of PAOmix, EUBmix, GB, GB_G1 and GB_G2, and appropriate competitors. The FISH probes and competitors used in this study are listed in Table 1.

FISH analyses. For FISH analyses of GB subgroups, sludge samples collected from the SBR at the end of the anaerobic phase were fixed with $4 \%$ paraformaldehyde/PBS $\left(137 \mathrm{mM} \mathrm{NaCl}, 8.1 \mathrm{mM} \mathrm{Na}_{2} \mathrm{HPO}_{4}\right.$, $2.68 \mathrm{mM} \mathrm{KCl}, 1.47 \mathrm{mM} \mathrm{KH}_{2} \mathrm{PO}_{4}$, pH 7.2) for $4 \mathrm{~h}$ at $4{ }^{\circ} \mathrm{C}$. Fixed sludge samples were washed twice in PBS, resuspended in PBS/ethanol $(1: 1$, $\mathrm{v} / \mathrm{v}$ ), and then stored at $-20{ }^{\circ} \mathrm{C}$ until FISH analyses. Fixed sludge samples were homogenized using a homogenizer (Dispergierantrieb Top10 basic; IKA, Germany) at $45 \mathrm{~s}$ for three cycles at maximum speed prior to the FISH experiments in order to remove cell aggregates. FISH experiments were performed on poly-L-lysine-coated slides (Sigma) 
Table 1. Information relevant to the FISH oligonucleotides designed and used in this study

\begin{tabular}{|c|c|c|c|c|}
\hline Probe name & Sequence $\left(5^{\prime}-3^{\prime}\right)$ & Target organisms & Competitors & Reference \\
\hline GB429* & ССССАССТAAAGGGCTTT & GB8 & GB429_C1 and 2 & This study \\
\hline GB429_C1† & CCCCGCCTAAAGGGCTTT & GB1-7 & & This study \\
\hline GB742* & CTCAGCGTCAGTGTGGCC & GB1-8 & & This study \\
\hline GB & CGATCCTCTAGCCCACT & GB1-7 & GB_C & Kong et al. (2002) \\
\hline GB_C $\dagger$ & CAGTCCTCTAGCCCACT & GB8 & & This study \\
\hline GB_G1_C $\dagger$ & TTCCCCAGATGTCAAGGC & & & Crocetti et al. (2002) \\
\hline GB_G2 & TTCCCCAGATGTCAAGGC & GB5 and 7 & GB_G2_C & Kong et al. (2002) \\
\hline GB_G2_C $\dagger$ & TTCCCCGGATGTCAAGGC & & & Kong et al. (2002) \\
\hline EUB338I\$ & GCTGCCTCCCGTAGGAGT & Eubacteria & & Amann et al. (1995) \\
\hline EUB338IIऽ & GCAGCCACCCGTAGGTGT & Eubacteria & & Daims et al. (1999) \\
\hline EUB338IIIs & GCTGCCACCCGTAGGTGT & Eubacteria & & Daims et al. (1999) \\
\hline
\end{tabular}

${ }^{*}$ rRNA, E. coli numbering in this study.

$\dagger$ Competitor probes are designated $\mathrm{C}$.

\$Probe GB_G1 was also named GAOQ989 (Crocetti et al., 2002).

§Used as EUBmix.

IIProbe NonEub338, complementary to Eub338, was used to exclude non-specific binding.

IUsed as PAOmix.

using previously described methods (Amann et al., 1995; Hugenholtz et al., 2002). Non-probe controls were prepared to examine autofluorescent bacteria and the NonEUB338 probe was used for nonspecific oligonucleotide binding (Amann et al., 1995). Slides for FISH analyses were mounted in Citifluor (Citifluor, UK) and viewed. A total of three images per specimen were captured at $\times 1000$ magnification using a Zeiss Axiophot epifluorescence microscope equipped with an HBO100 mercury vapour short-arc lamp, filter-sets no. 10 and no. 20 for fluorescein and $\mathrm{Cy} 3$, respectively, and an AxioCam MRm digital camera (Zeiss). Enhanced FISH images were obtained using AxioVs40 V 4.7.1.0 (Zeiss) by the combination of the three captured images, and Nomarski photographs were also collected simultaneous to the FISH images. The abundance of the probe-targeted cells was quantified using more than 20 FISH images per sample.

FISH/MAR analyses. To evaluate the abilities of GB8 to take up acetate and phosphorus, combined FISH and MAR experiments were carried out according to previously described procedures (Lee et al., 1999; Nielsen et al., 2003; Kong et al., 2004; Kim et al., 2010). Sludge samples collected from the SBR at the ends of the aerobic and anaerobic phases were anaerobically and aerobically incubated with sodium $\left[1-{ }^{14} \mathrm{C}\right]$ acetate (Amersham) and ${ }^{33} \mathrm{P}_{\mathrm{i}}$ (Perkin Elmer), each at a concentration of $10 \mu \mathrm{Ci}(370 \mathrm{kBq})$ per mg suspended solids (Lee et al., 1999; Kim et al., 2010). All anaerobic preparations of the sludge samples were carefully flushed with $\mathrm{O}_{2}$-free $\mathrm{N}_{2}$. For FISH/MAR, FISH hybridization methods were the same as those described for FISH analyses except for the use of coverslips instead of poly-L-lysinecoated slides. The subsequent steps of the MAR protocol were performed as detailed by Lee et al. (1999). KODAK type NTB was used as an autoradiographic liquid emulsion following the instructions of the manufacturer. For negative controls, sludge samples pasteurized at $70{ }^{\circ} \mathrm{C}$ for $10 \mathrm{~min}$ were incubated with radioactive and nonradioactive substrates in parallel in all experiments. All coverslips for FISH/MAR analyses were mounted in Citifluor and placed upside down. Views of probe-targeted cells were identified first under fluorescence excitation as described for FISH analyses, and then bright-view photographs of the same field using black silver particles for the MAR signals were collected.

\section{RESULTS AND DISCUSSION}

\section{Reactor operation}

An anaerobic/aerobic SBR for EBPR was continually operated for more than 8 months under the same conditions, supplied with sodium acetate as the sole carbon source. After initiation of the SBR by an inoculation of activated sludge seed, phosphate $\left(\mathrm{PO}_{4}-\mathrm{P}\right)$ release during the anaerobic phase and phosphate accumulation during the subsequent aerobic phase gradually increased with operation time. Complete $\mathrm{PO}_{4}-\mathrm{P}$ uptake at the end of the aerobic phase was accomplished after about 2 months of operation. After approximately 4 months of operation, soluble $\mathrm{PO}_{4}-\mathrm{P}$ values reached more than $90 \mathrm{mg} \mathrm{l}^{-1}$ at the end of the anaerobic phase. FISH analysis showed that cells (Accumulibacter) labelled with PAOmix probes constituted more than $55 \%$ of the total cells labelled with the EUBmix probes. However, after 6 months of operation, $\mathrm{PO}_{4}-\mathrm{P}$ release in the anaerobic phase decreased to below $55 \mathrm{mg}$ $1^{-1}$, and the population of Accumulibacter decreased to less than $25 \%$ of the total cells detected with EUBmix probes, although complete $\mathrm{PO}_{4}-\mathrm{P}$ uptake was still accomplished during the aerobic phase (data not shown). At this time, 
microscopic analysis showed that many cells with morphologies consistent with the Gram-negative large coccobacilli (2-4 $\mu \mathrm{m}$ in diameter) of GB were observed in the sludge (Crocetti et al., 2002; Kong et al., 2002). However, these cells did not show clear signals with the already known GBtargeting FISH probes in Table 1 (data not shown), suggesting that the EBPR sludge might contain previously unidentified members of GB. Therefore, subsequent $16 \mathrm{~S}$ rRNA gene sequencing and FISH/MAR analyses were performed for the phylogenetic determination and physiological characterization of the unidentified GB subgroup.

\section{Phylogenetic analysis of the new GB subgroup}

To analyse the phylogeny of the predicted new GB, a $16 \mathrm{~S}$ rRNA gene clone library was constructed using genomic DNA from a sludge sample containing cells with large coccobacilli morphologies. A total of 114 bacterial $16 \mathrm{~S}$ rRNA gene clones were selected randomly and were evaluated via RFLP analysis using HaeIII and HhaI double digestion of PCR amplicons. Representative clones (43 clones) with unique RFLP patterns were partially sequenced (approx. $700 \mathrm{nt}$ ), and the resulting sequences were submitted to GenBank for BLASTN searches. Accumulibacter-related 16S rRNA gene clones (nine of 43 representative clones) were equivalent to approximately $17.5 \%$ of the total clones (20 of 114 total clones) and Gammaproteobacteria-related $16 \mathrm{~S}$ rRNA gene clones (six of 43 representative clones) made up approximately $28.1 \%$ (32 of 114 clones) of the total clones. However, no $16 \mathrm{~S}$ rRNA gene sequence related to the genera Defluviicoccus and Sphingomonas of Alphaproteobaectria was identified from the clone libraries. The six representative 16S rRNA gene clones related to Gammaproteobacteria were further sequenced $(>1430 \mathrm{nt})$ and analysed phylogenetically. Among them, four representative $16 \mathrm{~S}$ rRNA gene clones making up approximately $21.9 \%$ (25 of 114) of the total clones were closely related to members of the known GB subgroups. The 16S rRNA gene sequences of GB retrieved from this study formed a clearly distinct phylogenetic lineage, designated GB8, from those of the known GB, namely GB1-GB7 (Kong et al., 2002), with relatively low sequence identities of 89.2 to $92.2 \%$, suggesting a novel putative subgroup of GB (Fig. 1). However, the phylogenetic analysis showed that the GB8 sequences of this study formed a phylogenetic lineage with some other 16S rRNA gene sequences (e.g. Jiang clones 71 and 91) from previous studies with high bootstrap values (99-100\%) (Fig. $1)$. Although they had relatively low sequence identities $(<97.0 \%)$, bacteria containing 16S rRNA gene sequences of Jiang clones 71 and 91 also might be members of the GB8 subgroup of Competibacter because the 16S rRNA gene sequences were retrieved from an anaerobic/aerobic cycled reactor for PHA production (Jiang et al., 2009). The maximum-likelihood tree also showed that clades of each GB subgroup comprised identical members of $16 \mathrm{~S}$ rRNA gene sequences to those of the neighbour-joining tree with basically the same topology (data not shown). Because the 16S rRNA gene sequences of GB8 were quite different from those of already known GB subgroups and they did not hybridize with any of the known GB-targeting probes (Kong et al., 2002), oligonucleotide FISH probes and competitors targeting the new GB subgroup (GB8) and all of the GB subgroups including GB8 were designed.

\section{FISH probe designs and evaluation}

To apply newly designed FISH probes to environmental samples, optimal conditions of binding stringency to avoid non-specific results should be determined; this is generally achieved by the use of controls such as cultured organisms or clones with zero or one mismatch within the rRNA probe targeting sequences (Hugenholtz et al., 2002; Schramm et al., 2002). However, this specificity of any single probe to the target group is not guaranteed since most of the target organisms are unculturable, and the environmental samples include a large proportion of unknown organisms. A solution to this limitation is the design and application of specific probes for multiple phylogenetic groups that encompass the phylogenetic hierarchies of the uncultured target group. Therefore, FISH probes targeting uncultured GB subgroups have been designed at different hierarchical levels and applied to sludge samples. We evaluated the specificities of existing GB FISH probes for the GB8 members and designed a GB8-specific FISH probe and a new FISH probe targeting all of the GB subgroups including GB8 for ecophysiological studies of GB8.

First, the specificities of the existing GB-targeting FISH probes in Table 1 were evaluated using the sludge samples containing the new GB subgroup, GB8. Analysis of $16 \mathrm{~S}$ rRNA gene sequences of all GB subgroups of Fig. 1 showed that known FISH probes targeting existing GB subgroups (GB1-GB7) at different hierarchical levels (Kong et al., 2002) did not adequately match with the $16 \mathrm{~S}$ rRNA gene sequences of GB8. However, our FISH experiments revealed that the FISH probe GB (Table 1), which targeted all existing GB subgroups (GB1-GB7), produced weak positive signals for the sludge samples even with $70 \%$ FA. These FISH signals generally overlapped with the GB429 FISH probe-targeted cells (GB8-specific FISH probe designed in this study; see the following results of the GB429 FISH probe design). There were two mismatches between the GB probe sequence (5'-CGATCCTCTAGCCCACT $-3^{\prime}$ ) and the GB probe-targeting positions (5'AGTGGGCTAGAGGACTG-3') of GB8 members in the comparisons of the GB probe sequence with the 16S rRNA gene sequences of GB8 members, suggesting that the weak positive signals of the FISH probe GB might be produced by members of GB8 in the sludge samples. Therefore, a GB competitor, GB_C (5'-CAGTCCTCTAGCCCACT-3'), was designed for GB probe-targeting sequences of $\mathrm{GB} 8$ members. Because optimal FA concentrations for the GB FISH probe were 65-70\% (Kong et al., 2002), FISH experiments were performed with the sludge samples with $70 \%$ FA, using the GB FISH probe with non-labelled GB_C. In these experiments the weak positive signals 


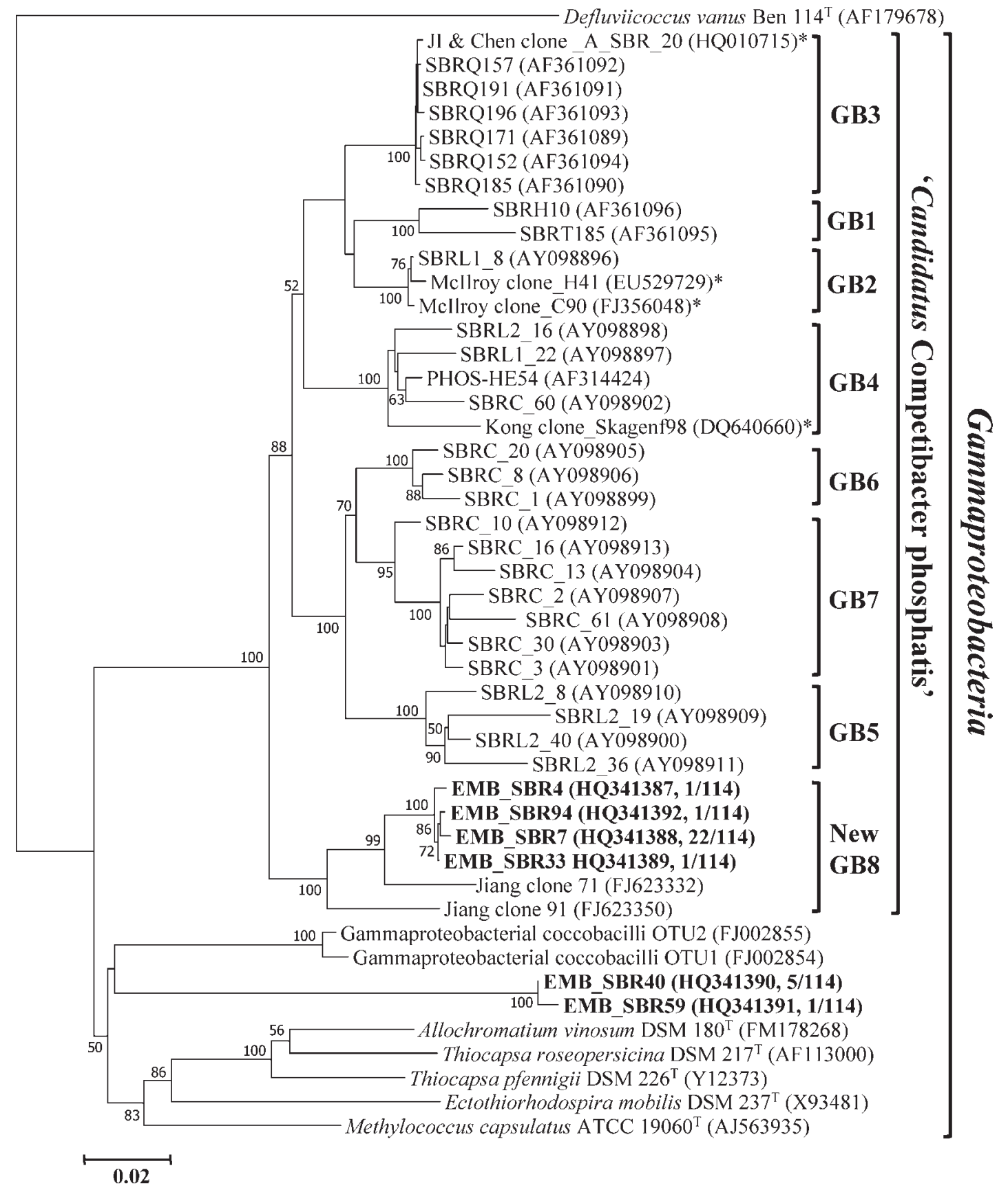

Fig. 1. Phylogenetic analysis of Gammaproteobacteria-related $16 \mathrm{~S}$ rRNA gene sequences from EBPR sludge. Sequences retrieved in this study (bold) were compared with those of reference sequences from GenBank. Bootstrap values $>50 \%$ are shown in percentages of 1000 replicates. Defluviicoccus vanus Ben $114^{\top}$ (AF179678) was used as an outgroup. The asterisks indicate 16S rRNA gene sequences that have been classified as members of the order Chromatiales of Gammaproteobacteria in 'RDP classifier'. The scale bar indicates the number of changes per nucleotide position.

disappeared, and only a few clear GB probe-positive signals were present, which suggests that the FISH probe GB with the GB competitor GB_C did not hybridize with GB8 members. The GB_G1 and GB_G2 probes of Table 1 were also tested with the sludge samples, but these probes did not produce positive signals for GB429-targeted cells (data not shown), indicating that the GB_G1 and GB_G2 probes have sufficient specificities for their target bacteria, at least with respect to the newly reported GB8 group.

A specific oligonucleotide FISH probe, GB429, which targeted members of GB8 was designed using the probe 
design tool in the ARB software package (Table 1), and was confirmed using the 'Probe Match' tool of the Ribosomal Database Project (Cole et al., 2009). An optimal FA concentration for the GB429 FISH probe for FISH analysis was determined using increments of FA for PFA-fixed sludge since no pure culture was available. The optimal FA concentration of the GB429 FISH probe was approximately $35 \%$. FISH analysis using the FISH probe GB429 always produced positive clear fluorescent signals as well as a small number of weak signals for our sludge samples (data not shown). Therefore, two sets of GB429 competitors, GB429_C1 and GB429_C1, which targeted the other seven GB subgroups (GB1-GB7) were also designed (Table 1). In FISH experiments using the GB429 FISH probe with two competitors, GB429_C1 and GB429_C2, the weak signals disappeared and only clear FISH signals were produced (Fig. 2). Probe GB429-targeted cells (members of GB8) were Gram-negative large coccobacilli about 2$4 \mu \mathrm{m}$ in size (Figs 2 and 3) and had morphological characteristics distinct from those of other EUBmixpositive cells (Fig. 2a).
A new FISH probe, GB742, which targets all of the GB subgroups, including members of GB8, was also designed. However, confirmation of the GB742 FISH probe by the 'Probe Match' tool of the Ribosomal Database Project indicated that the sequence of this probe matched several 16S rRNA gene sequences classified as the order Chromatiales of the Gammaproteobacteria. Phylogenetic analysis based on $16 \mathrm{~S}$ rRNA gene sequences indicated that these sequences are also members of GB subgroups that have been misclassified as Chromatiales sequences from other activated sludge samples (Fig. 1), indicating that the GB742 probe can target all GB subgroups of Compatibacter. Moreover, GB742-positive cells were completely matched with cells hybridized by GB+GB429 probe mixture (Fig. 2c), which also supported the sufficient specificity of the GB742 FISH probe. Members of the two more distantly related 16S rRNA gene sequences (FJ002855 and FJ002854) to the seven GB subgroups were also reported to show typical phenotypic properties of GB such as acetate uptake as PHA (Schroeder et al., 2009). However, they were excluded in the design of the GB742
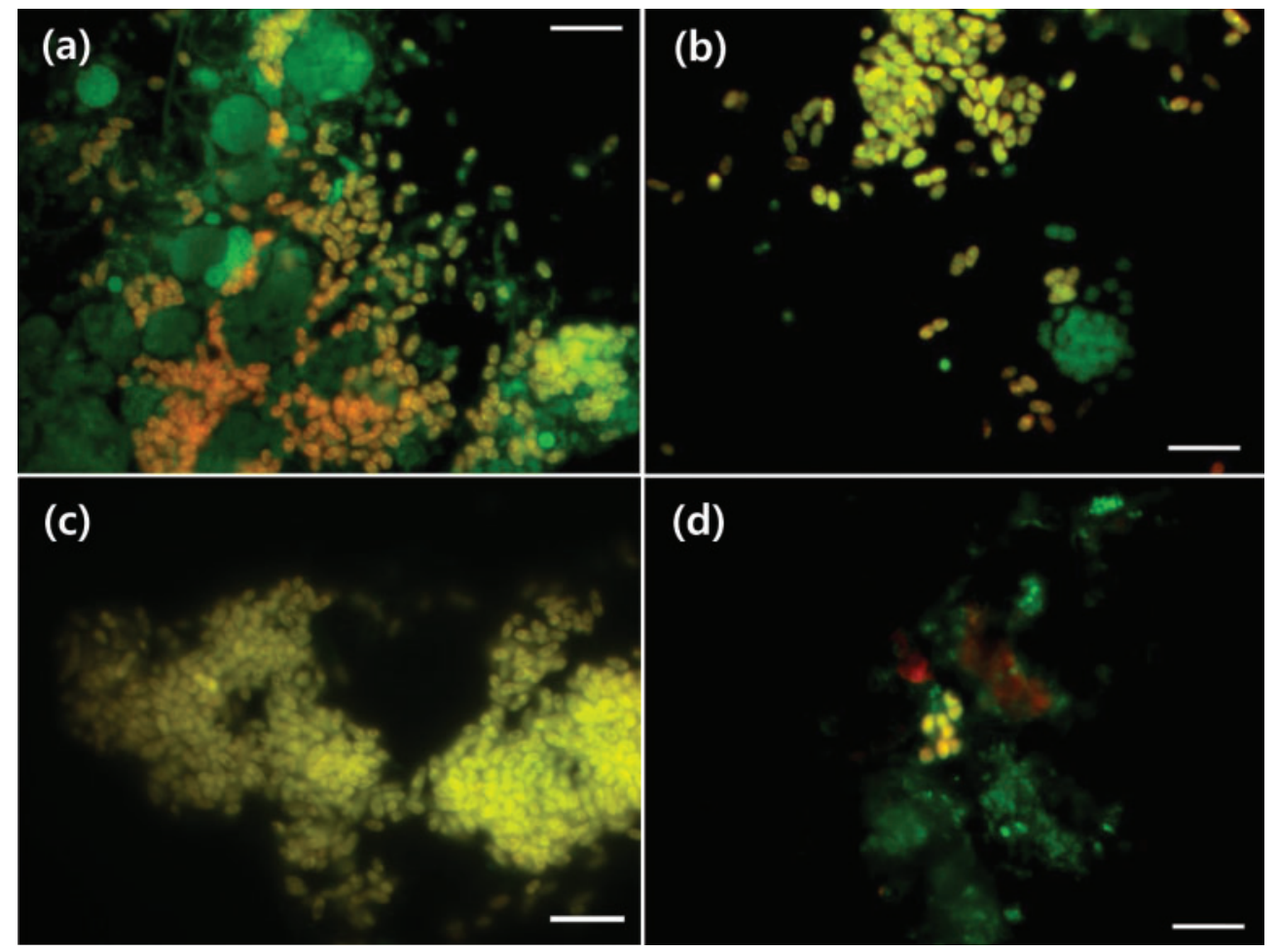

Fig. 2. FISH images of cells which hybridized with different FISH probes. (a) FISH images hybridized with GB429 (Cy3, red) and EUBmix (FITC, green). (b) FISH images hybridized with GB429 (Cy3, red) and GB742 (FITC, green) of lab-scale SBR sludge (from Kyungpook National University). The image indicates that the sludge sample contained members of other GB subgroups in addition to members of GB8. (c) FISH images hybridized with GB429 + GB (Cy3, red) and GB742 (FITC, green) of lab-scale SBR sludge (from Kyungpook National University). The image indicates that GB742-positive cells were completely matched with cells hybridized by the mixture of GB and GB429 probes. (d) FISH images hybridized with GB429 (Cy3, red) and EUBmix (FITC, green) of full-scale EBPR sludge (from Tancheon wastewater treatment plant). In all panels, yellow cells (overlay of red and green) indicate members of GB8 that were simultaneously hybridized with GB429 (Cy3, red) and other probes (FITC, green). Scale bars (all panels): $10 \mu \mathrm{m}$. 

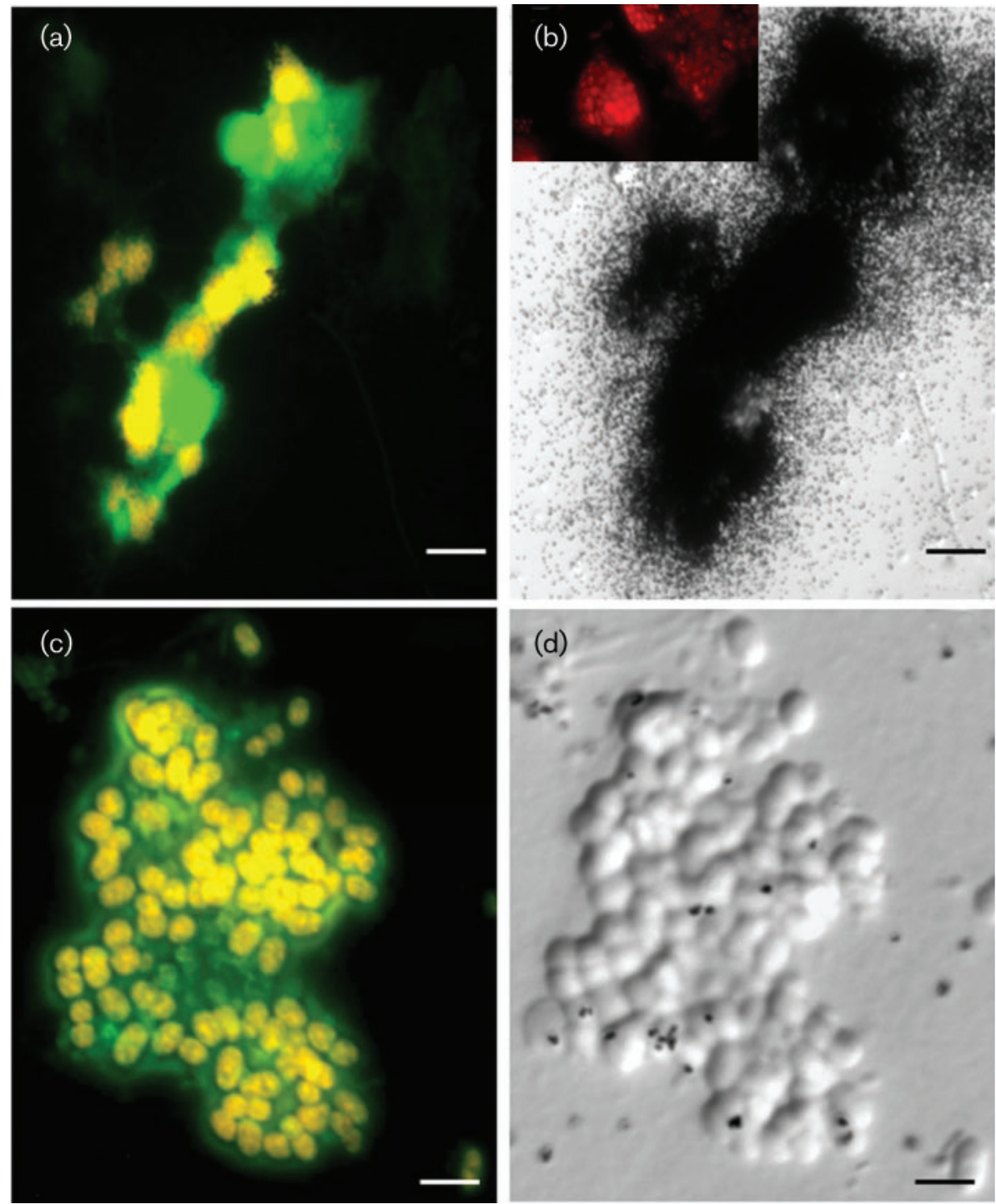

Fig. 3. FISH and MAR images of activated sludge. (a, c) FISH images showing cells hybridized with the combination of GB429 (Cy3, red) and EUBmix (FITC, green). Yellow cells (overlay of red and green) indicate members of the GB8 subgroup that were hybridized with both probes simultaneously. (b, d) Nomarski images show MAR-positive cells taking up $\left[{ }^{14} \mathrm{C}\right]$ acetate under anaerobic conditions (b) and MAR-negative cells with no uptake of ${ }^{33} \mathrm{P}_{\mathrm{i}}$ under aerobic conditions (d). The inset in (b) shows Nile blue staining of PHA. Scale bars: $20 \mu \mathrm{m}$ ( $a$ and b) and $5 \mu \mathrm{m}$ (c and d).

FISH probe which targeted all of the GB subgroups because they have not been detected in most EBPR plants or other activated-sludge processes, indicating that they may not be distributed widely in EBPR processes (Schroeder et al., 2009). The optimal FA concentration of the GB742 FISH probe was determined using increments of FA concentration for PFA-fixed sludge, and was found to be approximately $35 \%$. Although the target site of the GB742 probe was located in region IV of the Escherichia coli 16S rRNA gene sequence with relatively low accessibility (Fuchs et al., 2000), FISH hybridization using the GB742 probe yielded sufficiently strong fluorescent signals with a $35 \%$ FA concentration (Fig. 1), indicating that helper probes were not necessary for the increase of signal intensity.

\section{Ecophysiology analysis of GB8 using FISH/MAR analyses}

Complete $\mathrm{PO}_{4}-\mathrm{P}$ uptake at the end of the aerobic phase was accomplished during 8 months of SBR operation. However, FISH analyses showed that members of GB8 labelled with the GB429 FISH probe coexisted together with members of Accumulibacter in sludge samples that were labelled with PAOmix probes (data not shown), and that the population densities of GB8 (5-25\% of EUBmix- 
Table 2. Distribution of GB subgroups in sludge samples from two lab-scale reactors and three full-scale plants for EBPR

\begin{tabular}{|lccc|}
\hline $\begin{array}{l}\text { Sludge } \\
\text { sample }^{\star}\end{array}$ & $\begin{array}{r}\text { Sample } \\
\text { source } \dagger\end{array}$ & $\begin{array}{c}\text { GB-targeted } \\
\text { cells }(\text { GB1-7) }\end{array}$ & $\begin{array}{c}\text { GB429-targeted } \\
\dagger\end{array}$ \\
\hline CAU & cells $($ GB8) $(\%) \dagger$ \\
KNU & Lab-scale & 11.8 & 88.2 \\
Tancheon & Full-scale & 39.1 & 60.9 \\
POSTECH & Full-scale & 19.3 & 80.7 \\
Jungnang & Full-scale & 29.8 & 61.4 \\
& & & 70.1 \\
\hline
\end{tabular}

${ }^{\star} \mathrm{CAU}$ and KNU represent sludge samples from Chung-Ang University and Kyungpook National University, respectively.

$\dagger$ Percentages of cells targeted by each probe for all GB cells labelled with the GB742 probe.

binding cells) and Accumulibacter (25-55\% of EUBmixbinding cells) fluctuated greatly with operation time although the reactor operation was constant and showed good EBPR. With increases in GB429-targeted cell densities, both PAOmix-targeted cell densities and $\mathrm{PO}_{4}-\mathrm{P}$ release during the anaerobic phase decreased, suggesting that GB429-targeted cells competed with Accumulibacter for acetate uptake during the anaerobic phase.

FISH/MAR combinational analysis demonstrated that bacterial cells responding to the GB429 FISH probe (members of GB8) were able to take up acetate as PHA during the anaerobic phase (Fig. 3b), but they had no ability to accumulate orthophosphate during the subsequent aerobic phase (Fig. 3d), consistent with in situ phenotypic properties of GB in the biochemical model (Satoh et al., 1994; Liu et al., 1996; Jeon et al., 2001). Also, they out-competed Accumulibacter for acetate uptake during the anaerobic phase.

Additional FISH analysis on sludge samples taken from an SBR of another lab (Kyungpook National University, Korea) displaying EBPR failure showed that GB429targeted cells predominated ( $61 \%$ of total GB cells labelled with GB742 probe), suggesting that these cells could be important GAO. Subsequently, GB429 and GB742 probes were applied to several sludge samples from two other labscale and three full-scale EBPR processes. Probe GB429positive cells (members of GB8) were detected from all sludge samples in different cell densities $(5-25 \%$ at labscale SBR and $1-5 \%$ at full-scale EBPR plants), suggesting that members of GB8 might be widely distributed in EBPR processes (Fig. 2b, d). Probe GB742-positive cells (all GB) without hybridization signals of the GB429 FISH probe (GB8) were also detected (Fig. 2b), indicating that other members of the GB subgroups except for GB8 also existed in the sludge samples. The quantitative analysis of GB subgroups in sludge samples from two lab-scale reactors and three full-scale plants showed that the probe GB429positive cells (GB8) were more dominant than the probe GB-positive cells (GB1-7) in all sludge samples, as shown in Table 2, indicating that the GB8 subgroup might be more significant than subgroups GB1-7 in EBPR sludge of Korea.

\section{Conclusions}

The presence of GAO has been hypothesized to be a cause of deterioration in EBPR processes due to their ability to out-compete PAO (Accumulibacter). In this study, a new subgroup (GB8) of GB (Compatibacter) with a distinct phylogenetic lineage based on 16S rRNA gene sequences was discovered in SBR sludge. The subgroup showed morphological and physiological properties (Gramnegative large coccobacilli about $2-4 \mu \mathrm{m}$ in size, acetate uptake under anaerobic conditions without subsequent polyphosphate accumulation under aerobic conditions) consistent with those of previously reported GB. Because no existing GB-targeting FISH probes matched the $16 \mathrm{~S}$ rRNA gene sequences of GB8 members, a GB8-specific probe and a FISH probe which targeted all GB subgroups including GB8 were designed. FISH/MAR approaches showed that members of GB8 have typical properties of GAO with abilities to take up acetate under anaerobic conditions and without accumulation of polyphosphate under the subsequent aerobic conditions, and they were distributed widely in several lab- and full-scale EBPR processes as a significant GB subgroup. Therefore, designs of a set of FISH probes which hybridize all GB subgroups at different hierarchical levels will allow for monitoring of the distributions of GB and studies into their ecophysiologies in lab- or full-scale EBPR plants.

\section{ACKNOWLEDGEMENTS}

These efforts were supported by grants from the 21C Frontier Microbial Genomics and Application Center Program (grant no. MG05-0104-4-0) and the Next-Generation BioGreen 21 Program, Rural Development Administration, Republic of Korea (no. SSAC2011-PJ008220).

\section{REFERENCES}

Amann, R. I., Ludwig, W. \& Schleifer, K. H. (1995). Phylogenetic identification and in situ detection of individual microbial cells without cultivation. Microbiol Rev 59, 143-169.

Bond, P. L., Hugenholtz, P., Keller, J. \& Blackall, L. L. (1995). Bacterial community structures of phosphate-removing and non-phosphateremoving activated sludges from sequencing batch reactors. Appl Environ Microbiol 61, 1910-1916.

Burow, L. C., Kong, Y. H., Nielsen, J. L., Blackall, L. L. \& Nielsen, P. H. (2007). Abundance and ecophysiology of Defluviicoccus spp., glycogenaccumulating organisms in full-scale wastewater treatment processes. Microbiology 153, 178-185.

Cech, J. S. \& Hartman, P. (1993). Competition between polyphosphate and polysaccharide accumulating bacteria in enhanced biological phosphate removal systems. Water Res 27, 1219-1225.

Cole, J. R., Wang, Q., Cardenas, E., Fish, J., Chai, B., Farris, R. J., Kulam-Syed-Mohideen, A. S., McGarrell, D. M., Marsh, T. \& other authors (2009). The Ribosomal Database Project: improved alignments 
and new tools for rRNA analysis. Nucleic Acids Res 37 (Database issue), D141-D145.

Crocetti, G. R., Hugenholtz, P., Bond, P. L., Schuler, A., Keller, J., Jenkins, D. \& Blackall, L. L. (2000). Identification of polyphosphateaccumulating organisms and design of $16 \mathrm{~S}$ rRNA-directed probes for their detection and quantitation. Appl Environ Microbiol 66, 11751182 .

Crocetti, G. R., Banfield, J. F., Keller, J., Bond, P. L. \& Blackall, L. L. (2002). Glycogen-accumulating organisms in laboratory-scale and fullscale wastewater treatment processes. Microbiology 148, 3353-3364.

Daims, H., Brühl, A., Amann, R., Schleifer, K. H. \& Wagner, M. (1999). The domain-specific probe EUB338 is insufficient for the detection of all Bacteria: development and evaluation of a more comprehensive probe set. Syst Appl Microbiol 22, 434-444.

DeSantis, T. Z., Hugenholtz, P., Larsen, N., Rojas, M., Brodie, E. L., Keller, K., Huber, T., Dalevi, D., Hu, P. \& Andersen, G. L. (2006). Greengenes, a chimera-checked $16 \mathrm{~S}$ rRNA gene database and workbench compatible with ARB. Appl Environ Microbiol 72, 50695072.

Felsenstein, J. (2002). PHYLIP (phylogeny inference package), version 3.6a. Distributed by the author. Department of Genome Sciences, University of Washington, Seattle, USA.

Fuchs, B. M., Glöckner, F. O., Wulf, J. \& Amann, R. (2000). Unlabeled helper oligonucleotides increase the in situ accessibility to 16S rRNA of fluorescently labeled oligonucleotide probes. Appl Environ Microbiol 66, 3603-3607.

Hugenholtz, P., Tyson, G. W. \& Blackall, L. L. (2002). Design and evaluation of $16 \mathrm{~S}$ rRNA-targeted oligonucleotide probes for fluorescence in situ hybridization. Methods Mol Biol 179, 29-42.

Jeon, C. O. \& Park, J. M. (2000). Enhanced biological phosphorus removal in a sequencing batch reactor supplied with glucose as a sole carbon source. Water Res 34, 2160-2170.

Jeon, C. O., Lee, D. S., Lee, M. W. \& Park, J. M. (2001). Enhanced biological phosphorus removal in an anaerobic-aerobic sequencing batch reactor: effect of pH. Water Environ Res 73, 301-306.

Jeon, C. O., Lee, D. S. \& Park, J. M. (2003a). Microbial communities in activated sludge performing enhanced biological phosphorus removal in a sequencing batch reactor. Water Res 37, 2195-2205.

Jeon, C. O., Park, W., Padmanabhan, P., DeRito, C., Snape, J. R. \& Madsen, E. L. (2003b). Discovery of a bacterium, with distinctive dioxygenase, that is responsible for in situ biodegradation in contaminated sediment. Proc Natl Acad Sci U S A 100, 13591-13596.

Jiang, Y., Chen, Y. \& Zheng, X. (2009). Efficient polyhydroxyalkanoates production from a waste-activated sludge alkaline fermentation liquid by activated sludge submitted to the aerobic feeding and discharge process. Environ Sci Technol 43, 7734-7741.

Kim, J. M., Le, N. T., Chung, B. S., Park, J. H., Bae, J. W., Madsen, E. L. \& Jeon, C. O. (2008). Influence of soil components on the biodegradation of benzene, toluene, ethylbenzene, and $o_{-}^{-}, m_{-}$, and $p$-xylenes by the newly isolated bacterium Pseudoxanthomonas spadix BD-a59. Appl Environ Microbiol 74, 7313-7320.

Kim, J. M., Lee, H. J., Kim, S. Y., Song, J. J., Park, W. J. \& Jeon, C. O. (2010). Analysis of the fine-scale population structure of "Candidatus Accumulibacter phosphatis" in enhanced biological phosphorus removal sludge, using fluorescence in situ hybridization and flow cytometric sorting. Appl Environ Microbiol 76, 3825-3835.

Kong, Y. H., Ong, S. L., Ng, W. J. \& Liu, W. T. (2002). Diversity and distribution of a deeply branched novel proteobacterial group found in anaerobic-aerobic activated sludge processes. Environ Microbiol 4, 753-757.

Kong, Y. H., Nielsen, J. L. \& Nielsen, P. H. (2004). Microautoradiographic study of Rhodocyclus-related polyphosphate- accumulating bacteria in full-scale enhanced biological phosphorus removal plants. Appl Environ Microbiol 70, 5383-5390.

Kong, Y. H., Xia, Y., Nielsen, J. L. \& Nielsen, P. H. (2006). Ecophysiology of a group of uncultured gammaproteobacterial glycogen-accumulating organisms in full-scale enhanced biological phosphorus removal wastewater treatment plants. Environ Microbiol 8, 479-489.

Lane, D. J. (1991). 16S/23S rRNA sequencing. In Nucleic Acid Techniques in Bacterial Systematics, pp. 115-175. Edited by E. Stackebrandt \& M. Goodfellow. Chichester: Wiley.

Lee, N., Nielsen, P. H., Andreasen, K. H., Juretschko, S., Nielsen, J. L., Schleifer, K. H. \& Wagner, M. (1999). Combination of fluorescent in situ hybridization and microautoradiography - a new tool for structure-function analyses in microbial ecology. Appl Environ Microbiol 65, 1289-1297.

Liu, W. T., Mino, T., Nakamura, K. \& Matsuo, T. (1994). Role of glycogen in acetate uptake and polyhydroxyalkanoate synthesis in anaerobic-aerobic activated-sludge with a minimized polyphosphate content. J Ferment Bioeng 77, 535-540.

Liu, W. T., Mino, T., Nakamura, K. \& Matsuo, T. (1996). Glycogen accumulating population and its anaerobic substrate uptake in anaerobic-aerobic activated sludge without biological phosphorus removal. Water Res 30, 75-82.

Lu, S., Park, M. J., Ro, H. S., Lee, D. S., Park, W. J. \& Jeon, C. O. (2006). Analysis of microbial communities using culture-dependent and culture-independent approaches in an anaerobic/aerobic SBR reactor. J Microbiol 44, 155-161.

Ludwig, W., Strunk, O., Westram, R., Richter, L., Meier, H., Yadhukumar, Buchner, A., Lai, T., Steppi, S. \& other authors (2004). ARB: a software environment for sequence data. Nucleic Acids Res 32, 1363-1371.

Mcllroy, S. J., Nittami, T., Seviour, E. M. \& Seviour, R. J. (2010). Filamentous members of cluster III Defluviicoccus have the in situ phenotype expected of a glycogen-accumulating organism in activated sludge. FEMS Microbiol Ecol 74, 248-256.

Meyer, R. L., Saunders, A. M. \& Blackall, L. L. (2006). Putative glycogen-accumulating organisms belonging to the Alphaproteobacteria identified through rRNA-based stable isotope probing. Microbiology 152, 419-429.

Mino, T., Arun, V., Tsuzuki, Y. \& Matsuo, T. (1987). Effect of phosphorus accumulation on acetate metabolism in the biological phosphorus removal process. In Biological Phosphate Removal from Wastewaters, pp. 27-38. Edited by R. Ramadori. Oxford: Pergamon Press.

Mino, T., Liu, W. T., Kurisu, F. \& Matsuo, T. (1995). Modeling glycogen storage and denitrification capability of microorganisms in enhanced biological phosphate removal processes. Water Sci Technol 31, 25-34.

Mino, T., Van Loosdrecht, M. C. M. \& Heijnen, J. J. (1998). Microbiology and biochemistry of the enhanced biological phosphate removal process. Water Res 32, 3193-3207.

Nielsen, A. T., Liu, W. T., Filipe, C., Grady, L., Jr, Molin, S. \& Stahl, D. A. (1999). Identification of a novel group of bacteria in sludge from a deteriorated biological phosphorus removal reactor. Appl Environ Microbiol 65, 1251-1258.

Nielsen, J. L., Christensen, D., Kloppenborg, M. \& Nielsen, P. H. (2003). Quantification of cell-specific substrate uptake by probedefined bacteria under in situ conditions by microautoradiography and fluorescence in situ hybridization. Environ Microbiol 5, 202-211.

Oehmen, A., Lemos, P. C., Carvalho, G., Yuan, Z., Keller, J., Blackall, L. L. \& Reis, M. A. M. (2007). Advances in enhanced biological phosphorus removal: from micro to macro scale. Water Res 41, 2271-2300.

Pruesse, E., Quast, C., Knittel, K., Fuchs, B. M., Ludwig, W. G., Peplies, J. \& Glöckner, F. O. (2007). SILVA: a comprehensive online 
resource for quality checked and aligned ribosomal RNA sequence data compatible with ARB. Nucleic Acids Res 35, 7188-7196.

Satoh, H., Mino, T. \& Matsuo, T. (1994). Deterioration of enhanced biological phosphorus removal by the domination of microorganisms without polyphosphate accumulation. Water Sci Technol 30, 203-211.

Schramm, A., Fuchs, B. M., Nielsen, J. L., Tonolla, M. \& Stahl, D. A. (2002). Fluorescence in situ hybridization of 16S rRNA gene clones (Clone-FISH) for probe validation and screening of clone libraries. Environ Microbiol 4, 713-720.

Schroeder, S., Petrovski, S., Campbell, B., Mcllroy, S. \& Seviour, R. (2009). Phylogeny and in situ identification of a novel gammaproteobacterium in activated sludge. FEMS Microbiol Lett 297, 157-163.

Seviour, R. J. \& Mcllroy, S. (2008). The microbiology of phosphorus removal in activated sludge processes - the current state of play. J Microbiol 46, 115-124.
Seviour, R. J., Mino, T. \& Onuki, M. (2003). The microbiology of biological phosphorus removal in activated sludge systems. FEMS Microbiol Rev 27, 99-127.

Stamatakis, A., Ott, M. \& Ludwig, T. (2005). RAxML-OMP: an efficient program for phylogenetic inference on SMPs. Lect Notes Comput Sci 3506, 288-302.

Wang, Q., Shao, Y., Huong, V. T. T., Park, W. J., Park, J. M. \& Jeon, C. O. (2008). Fine-scale population structure of Accumulibacter phosphatis in enhanced biological phosphorus removal sludge. J Microbiol Biotechnol 18, 1290-1297.

Wong, M.-T., Tan, F. M., Ng, W. J. \& Liu, W.-T. (2004). Identification and occurrence of tetrad-forming Alphaproteobacteria in anaerobic-aerobic activated sludge processes. Microbiology 150, 3741-3748.

Edited by: H. Daims 